\title{
Protocols in Mobile Electronic Commerce
}

\author{
Mr. Vivek B. Patil, Mr.Deepak G. Awate \\ 'Research Student, Asso.Prof. Computer Science
}

\section{ABSTRACT}

Mobile-commerce, also known by other terms such as M-Commerce or m-Commerce, is the capability to follow commerce with the help of a hand held devise like mobile phone, a Personal digital assistant PDA, a smart phone, or other emerging mobile equipment such as dash top mobile devices. Since decade Mobile-commerce is being expressed as follows: Mobile-commerce is where any transaction, involving the transfer of rights or ownership to use goods and services, which is initialized and/or processed by using mobile (cell) access to computer-mediated (eg. WAN) networks with the help of an electronic device.

\section{INTRODUCTION}

E-Commerce is brief kind for electronic commerce and focuses to the sphere of promoting, purchasing, selling, servicing and distributing various products and/or services on the internet. It targets electronic business applications for the aim of commercial transactions. The quantity of trade conducted digitally has varied with widespread web usage. The activities of commerce is conducted in this way, prodding and drawing on innovations in electronic funds transfer, offer chain management, web selling, on-line dealings process, Electronic information Interchange (EDI), automatic information assortment systems and inventory management systems. Modernized electronic commerce uses the World Wide Web (Also known as the internet) at least at some point of time in the transaction's phase, even though it can utilizes a large range of technologies such as e-mail.

Online shopping is a one of the form of electronic commerce wherever the customer is directly on-line to the seller's PC sometimes via the net. There's no mediator service. The purchase and sale of products (transaction) is completed with the help of internet interactively in real-time such as advertising, selling of books on Amazon.com.

\section{CURRENT ERA WIRELESS MOBILE SYSTEM}

Wireless communications inculpate transmitting signals through air, water and space via radio waves. Wireless spectrum is allotted by governments and international organizations. For instance, within the United State the $800-\mathrm{MHz}$ frequency is allotted for cellular voice communications. The upper the frequency, the bigger the information measure and information carrying capability of the wireless system.
However, as frequency is increased in every system, its characteristics become more directional (line of site) and therefore the signals more vulnerable to atmospheric conditions like rain and fog. Wireless technologies are used to build LANs with in limited geographical areas like connecting two or more building or LANs. They're conjointly used to provide high-speed access to the net or to create metropolitan area networks. Wireless mobile systems with terribly high data-rates emerging that permit mobile users to work as if they're hooked up to traditional wired networks.

\section{MOBILE WIRELESS COMMUNICATIONS (GLOBAL SYSTEM FOR MOBILE)}

GSM might be a complicated cell framework developed by bunch extraordinary remote of the class Europeans Des Posts Telecommunications and its successor European Telecommunications Institute. An essential objective of the Global System for Mobile advancement methodology was to produce similarity of cell administrations between European countries. GSM can be a revolutionary deftness that blends Time Division Multiple Access.

The GSM improvement strategy was similar as that of AMPS. The house edges of the GSM remote plot from all vendors were waived, imagining gigantically far-famed.

\section{Electronic Commerce and Mobile commerce}

The meaning and conventions of electronic commerce have changed during past 31 years. Initially, electronic commerce was meant to, facilitation of all commercial transactional activities with the help of technology such as Electronic Funds Transfer (EFT) and Electronic Data Interchange (EDI). Both EDI and EFT were introduced in 1970s (2), facilitated businesses to 
send commercial instruments like purchase orders(PO) or invoices electronically.

\section{Mobile-commerce Transaction Model}

The recent cellular phones have plenty of extra capabilities compared to the older ones. Consequently, the telephone companies can offer plenty of so-called value-added services. Percentage of users for such services is foretold to extend because with new technologies new (and better) services are offered. In the future, many new services are expected. The flexibleness to pay by phone is one among such services, which is attention-grabbing to numerous corporations are interested. For example in country Finland, one is already able to make payment for the certain product by using their cell phone. Sending and receiving multimedia contents are also gaining interest. Since the entire internet wireless network is more on data-oriented, net will become a serious possibility as well. Mobile devices, which provide service not just for regular users however conjointly for mobile applications computer code developers, square measure of nice interest for the phone corporations.

\section{Wireless payment services}

Banks begin with large benefits in $\mathrm{m}$ commerce services like ticketing, auctions, and added information: They control the present payment infrastructure and understand the protection problems. The Pepsi Cola slot machine piloted by Finnish mobile-security specialist Sonera Smart Trust may be a hanging example of another use for mobile phones. The company's service permits the user to shop for a drink from a slot machine by dialing its variety on a portable device. Bluetooth, the rising commonplace for short-range radio transmissions, can build it even easier for customers to create little payments through mobile devices. Information Technology Research Institute Mobile e-commerce dealings model five. A user might, for instance, buy groceries by debiting from a card or a bank account. Support for such services couldn't only give a replacement revenue stream for banks however also cut back the number of money they handle.

The WAP Payment Service permits a merchant to get bill payment via WAP. By using such outstanding service merchants may avoid problems in taking care of their own WAP payment servers. Users of that service are merchants, which sell product or services on-line using WAP, therefore, needs an extensive payment resolution. The various WAP payment solutions, international and local payment methods, multiple credit cards are often supported by such service. When new payment methodology appears, it can be integrated into the payment service. Service should also be protected by using state of the art firewalls, encrypted databases and everyone the logical protection to make sure a high safety level. Service takes care also of administration and reconciliation of all transactions. methods. Immediately after each payment, whether or not it's been thriving or not, the businessperson is notified mechanically by the service. This notification is often sent using various methods and platforms. At any given point of time, merchant has an access to his shop's payment history through a secure internet interface device. Once a consumer has chosen the specified product with merchant's WAP store and is prepared to pay, merchant web redirects him to the WAP payment getway page to process his request. Here, the consumer can opt for an appropriate payment method and make the payment. The payment is processed directly by service.

Properties of the mobile e-commerce transaction model(s)

1. Relationship with environments previously analyzed

The mobile e-commerce environment is rather similar to the international banking environment, for which the S-transaction model was developed. In fact, the banking environment is a (hidden) part of the mobile e-commerce environment in some cases. Shortly, the common features are:

$>$ Both are following distributed and environments with autonomous players;

$>$ In both cases economic values are transferred;

$>$ In both cases the primarytarget of the transactions is to assure atomicity of the executions; in banking it is the money atomicity, in mobile e-commerce the certified delivery. This leads to compensation which is problematic in both cases. Similar considerations concerning the programs;

$>$ In both cases the durability and serializability are of similar importance and are solved at the same level in the system architecture (at the database level syntactically);

$>$ The same modelling ideas can be used in both environments.

$>$ There are some differences:

$>$ In the mobile e-commerce environment also complicated real actions such as physical goods delivery are included; these are missing from the international banking environment;

$>$ The mobile e-commerce environment is hostile in the sense that the customers or merchants might be traitorous which is not the case within the banking environment

$>$ The terminals of the international banking environment are very well secured against unauthorised use whereas in the mobile e- 
commerce environment a terminal can be easily stolen and can lead to an unauthorised use

$>$ The terminals in the mobile electronic commerce have much less processor, memory etc. resources than the terminals in international banking

$>$ The security mechanisms are different

$>$ In international banking there is one big trusted "middle-player" (SWIFT; www.swift.com) offering messaging services and other services whereas in the e-commerce this is not (yet) the case

$>$ The transaction scopes are different (reflected in the TIDs; there is not a global TID in the mobile e-commerce case, whereas in international banking transaction there is a global TID)

$>$ In S-transaction model it is reasonable to assume that all the global programs belonging to the S-transaction environment run the same request-response-ack/nack protocol, in the mobile e-commerce environment this is not clear. The reason is that there is an existing infrastructure and this is not homogeneous, but rather different from country to country. Neither can it be made homogeneous by adding the mobile e-commerce channel;

$>$ "Location invalidation", i.e., transaction becoming invalid because of out-dated location information is specific to wireless, nomadic environment and thus has not been considered in banking contexts.

2 The scope of the mobile e-commerce transactions The primary purpose of the transactional mechanisms is to guarantee the money atomicity, goods atomicity and at the end the certified delivery. When looking into the business cases, one sees immediately that there is not one a single point of control that could be allocated the responsibility to guarantee this in the same manner as in a conventional environment the $2 \mathrm{PC}$ client runs the $2 \mathrm{PC}$ protocol and guarantees that all subtransactions started at the diverse sites either commit or abort. Rather, each autonomous player has a partial responsibility here. So they start different transactions that are, however, interdependent, and moreover, partially subordinate. For instance, the contact with the credit card Company in business case 1 only follows after the customer has placed an order. Second, the delivery is only started if the merchant has become assured that he/she will get the funds for the goods ordered. Thus, the delivery is conditioned on the outcome of the payment.

What is common to all these cases is the fact that the customer is the ultimate origin of the interactions. Thus, we can in a natural way assume that the client is the entity being the root in an execution hierarchy.
The cases then differ in number of other layers the customer must take direct contact with - and thus run a concrete protocol with the player. Case by case it seem as follows.

There are, however, two types of protocols run at the customer terminal:

$>$ The actual ordering of the goods (embedded in request-response type of protocol, place order confirm/deny order)

$>$ Identification of the customer to the merchant (embedded into several request-response pairs), optional

$>$ There are four different kinds of protocols run at the client terminal:

$>$ The actual ordering of the goods (imbedded in request-response type of protocol, place order confirm/deny order)

$>$ Identification of the customer to the merchant (embedded into several request- response pairs), optional

$>$ Payment protocol against the payment service or against the bank (embedded into several request-response pairs)

$>$ Identification of the customer to the payment service (embedded into request-response pair)

Customer interacts only with merchant (MNO) requesting a service/contents (embedded into request-response pair)

In Figure a simplified view of the overall transaction structure in the cases where payment is implicit is given. The circle within which the components are described models the atomicity sphere of the transaction. The payment and delivery are sub transactions of controlled by the Merchant. The customer is at the root of the transaction tree.

The picture abstracts from the identification step, because it can be excluded from the scope of the mobile e-commerce transaction (i.e., it is step similar to scanning of the catalogues that do not need to be included within the transactional boundaries). The figure also abstracts from the possible cancellation of the order. It could be modeled as a sub transaction at the merchant that waits for the possible return of the goods and starts the reverse pay

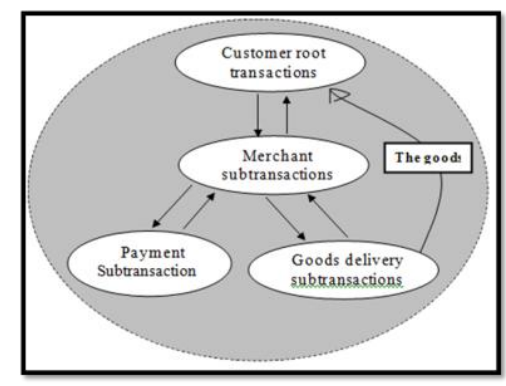

Figure Hierarchical views on the m-commerce transactions in cusses 


\section{OBJECTIVES AND SIGNIFICANCE OF STUDY}

A large percentage of transactions are conducted through mobile devices for virtual items and services such as access to premium and regular contents. The transaction; selling, purchasing and distributing services or /and product involves use of network and internet along additional communication devices which generally has low processing power, very low battery life , memory, intermittent connectivity and communication channel used along their bandwidth and also the security problems associated with. This research study is consecutive objectives;

$>$ Discuss the basic characteristics and attributes of e-commerce and m-commerce and protocols used at various stages in e-commerce.

$>$ Discuss m-commerce applications

$>$ Understand the technologies that support mobile communication system

$>$ To critically analyze protocols in m-commerce for fair-exchange, secure payment, automatic dispute resolving.

$>$ Protocols security in fixed Verses Wireless Communication

$>$ Performance of m-commerce protocols.

\section{CONCLUSION}

Mobile E-Commerce could be a difficult area as it creates opportunities for several players in the field, like Content and service providers or Mobile Network Operators. MEC is a dynamically ever-changing area then are the applications and needs due to the various interdependencies with user requirements, wireless technology and legislation. It's so essential that related business and legal problems are resolved and follow advances in technology.

\section{REFERENCE}

[1]. N. Asokan, 1998, "Fairness in Electronic Commerce", CCS '15 Proceedings of the twenty-second ACM SIGSAC Conference on pc and Communications Security, $\hat{A} 2015$.

[2]. Dhingra Kush, Bhardwaj Abhishek, Aashish Aggarwal (2015), M -Commerce , International Journal of Engineering Research and General Science, Volume 3, Issue 2, Part 2 , ISSN : ISSN 2091 - 2730 ,Page 406-706

[3]. K. R. Santhi,G. SenthilKumaran, V. K. Srivastava, A. Butare, "Goals of true broad bands wireless next wave (4G and 5G)" ,Vehicular Technology Conference-2003", VTC 2003-Fall, 2003 IEEE 58th, Volume: 4, 6-9 Oct. 2003.

[4]. J. Claessens, "Analysis and design of an advanced Infrastructure for Secure and
Anonymous Electronic Payment Systems on the internet", $\mathrm{PhD}$ thesis, Katholieke Universiteit Leuven, 2002, Page 220

[5]. T. Dierks, "The TLS Protocol Version 1.1", IETF internet Draft, 2003.

[6]. A. Alaraj and M. Munro, "An efficient ecommerce fair exchange protocol that encourages customer and merchant to be honest," computer Safety, reliability, and Security, pp. 193-206, 2008.

[7]. H. Aldabbas, T. Alwada'n, H. Janicke and A. Al-Bayatti, "Data Confidentiality in Mobile ad hoc Networks", International Journal of Wireless \& Mobile Networks (IJWMN), vol. 4, no. 1, february 2012. 